\title{
Salmonella bacteraemia in England and Wales, 1981-1990
}

\author{
E J Threlfall, M L M Hall, B Rowe
}

\begin{abstract}
Aims: To report the incidence of nontyphoidal salmonellas in England and Wales and identified in the Division of Enteric Pathogens, London between 1981 and 1990.

Methods: Strains were serotyped and phage typed for Salmonella typhimurium, $S$ enteritidis, and $S$ virchow, using established methods.

Results: Overall, less than $2 \%$ of nontyphoidal salmonellas isolated from humans were from blood culture. The highest numbers of bloodstream isolates were from infections caused by $S$ enteritidis and $S$ typhimurium, but the highest incidence of septicaemias was attributable to infections with $S$ cholerae-suis, $S$ dublin, and $S$ virchow. $2.2 \%$ of $S$ typhimurium isolates phage type 204C were from blood culture; likewise, $5.5 \%$ of $S$ virchow phage type 19. This could be a cause for concern as most isolates of both these phage types are multiresistant to antimicrobial drugs.

Conclusions: Salmonella septicaemia is rare in England and Wales in other than a few serotypes of limited epidemiological importance.
\end{abstract}

Salmonella septicaemia can be a lifethreatening disease and while it is commonly associated with Salmonella typhi, $S$ paratyphi $A$, and $S$ paratyphi $B$, it is uncommon for other serotypes. The Division of Enteric Pathogens (DEP) provides a reference service for salmonellas isolated from humans in England and Wales, and from 1981 to 1990 , 186244 non-typhoidal isolates belonging to 305 serotypes were identified. Although the most common symptomology was gastrointestinal, bloodstream invasion occurred in isolates from over 100 of these serotypes.

Division of Enteric Pathogens, Central Public Health Laboratory, 61 Colindale Avenue, London NW9 5HT E J Threlfall M L M Hall B Rowe

Correspondence to: E J Threlfall

Accepted for publication 4 July 1991

\section{Methods}

Strains were serotyped by the methods of Kauffman ${ }^{1}$ and phage typed by the methods of Callow $^{2}$ for $S$ typhimurium, by those of Ward $e t a \beta$ for $S$ enteritidis, and by those of Chambers et al for $S$ virchow.

Results

Of the 186244 isolates identified during the 10 year period 1981-1990, 2842 were from blood culture, an overall incidence of $1.5 \%$ (table 1 ). For the three most common serotypes, the incidence of blood infections was between $1 \%$ and $2 \%$ for $S$ enteritidis and $S$ typhimurium and $4 \%$ for $S$ virchow. In terms of the number of bloodstream isolates/isolates received, the highest incidence of blood infection was in $S$ cholerae-suis $(74 \%)$ followed by $S$ dublin (25\%). Only 27 isolates of $S$ cholerae-suis however, were identified and $S$ dublin was also an uncommon serotype, with only 430 isolates from humans over the study period. Other serotypes in which the incidence of blood infection was greater than $2 \%$ included $S$ bovis-morbificans, $S$ ealing, $S$ heidelberg, $S$ kedougou and $S$ panama.

For $S$ enteritidis the highest incidence of septicaemia (4.5\%) was associated with isolates which did not conform to recognisable phage type patterns (NC) (table 2). The epidemiological importance of this is difficult to interpret, however, as these isolates comprised strains with several different phage reaction patterns. In phage types of epidemiological importance the highest incidence of septicaemia ranged from $1.0 \%$ (phage type 6) to $3.4 \%$ (phage type 13a).

For $S$ typhimurium phage types the incidence of septicaemia ranged from $0.5 \%$ (phage type 49 ) to $2 \cdot 2 \%$ (phage type 204c). Previous studies have shown that all strains of phage type 204c are resistant to at least four antimicrobial drugs, including ampicillin, chloramphenicol, and trimethoprim. ${ }^{5}$

The $S$ virchow phage typing scheme has been in use since 1985 . Over the six years 1985-1990 the incidence of bloodstream invasion for phage types of this serotype

Table 1 Salmonellas from blood, England and Wales, 1981-90

\begin{tabular}{lrr}
\hline & $\begin{array}{l}\text { Total } \\
\text { No of } \\
\text { isolates }\end{array}$ & $\begin{array}{l}\text { Blood } \\
\text { isolates } \\
\text { No }(\%) \star\end{array}$ \\
\hline Serotype & 70498 & $981(1 \cdot 4)$ \\
S enteritidis & 64563 & $729(1 \cdot 1)$ \\
S typhimurium & 10554 & $406(3 \cdot 8)$ \\
S virchow & 430 & $109(25 \cdot 3)$ \\
S dublin & 1898 & $63(3 \cdot 3)$ \\
S heidelberg & 1756 & $48(2 \cdot 7)$ \\
S panama & 945 & $21(2 \cdot 2)$ \\
S kedougou & 27 & $20(74 \cdot 1)$ \\
S cholerae-suis & 321 & $7(2 \cdot 2)$ \\
S bovis-morbificans & 261 & $6(2 \cdot 3)$ \\
S ealing & 34991 & $452(1 \cdot 3)$ \\
Others (295 serotypes) & 186244 & $2842(1 \cdot 5)$ \\
Totals & &
\end{tabular}

*Percentage of each serotype. 
Table 2 Blood isolations in phage types of $S$ enteritidis, $S$ typhimurium, and $S$ virchow 1981-1990

\begin{tabular}{|c|c|c|c|c|c|c|c|c|}
\hline \multicolumn{3}{|l|}{$S$ enteritidis } & \multicolumn{3}{|c|}{ S typhimurium } & \multicolumn{3}{|l|}{$S$ virchow ${ }^{\star}$} \\
\hline Phage type & Total & Blood No $(\%) \dagger$ & Phage type & Total & Blood No (\%) & Phage type & Total & Blood No (\%) \\
\hline $\begin{array}{l}4 \\
8 \\
1 \\
6 \\
13 \mathrm{a} \\
\mathrm{NC} \\
11 \\
2 \\
\text { Others }\end{array}$ & $\begin{array}{r}54307 \\
6275 \\
1412 \\
2693 \\
679 \\
470 \\
671 \\
246 \\
3745\end{array}$ & $\begin{array}{r}720(1.3) \\
104(1.7) \\
27(1.9) \\
26(1.0) \\
23(3.4) \\
21(4.5) \\
12(1.8) \\
9(3.6) \\
39(1.0)\end{array}$ & $\begin{array}{c}12 \\
193 \\
49 \\
204 \mathrm{c} \\
204 \\
104 \\
141 \\
\text { NC } \\
49 \mathrm{a} \\
170 \\
\text { Others }\end{array}$ & $\begin{array}{r}7699 \\
4224 \\
8325 \\
1786 \\
3049 \\
4092 \\
3275 \\
1669 \\
2866 \\
3416 \\
24170 \\
64571\end{array}$ & $\begin{array}{r}123(1 \cdot 6) \\
61(1 \cdot 4) \\
44(0 \cdot 5) \\
40(2 \cdot 2) \\
39(1 \cdot 3) \\
34(0 \cdot 8) \\
33(1 \cdot 0) \\
27(1 \cdot 6) \\
25(1 \cdot 6) \\
21(0 \cdot 6) \\
282(1 \cdot 2) \\
729(1 \cdot 1)\end{array}$ & $\begin{array}{c}8 \\
19 \\
31 \\
26 \\
21 \\
2 \\
\text { Others }\end{array}$ & $\begin{array}{r}1087 \\
475 \\
921 \\
866 \\
504 \\
295 \\
2079\end{array}$ & $\begin{array}{r}91(8 \cdot 4) \\
26(5 \cdot 5) \\
22(2 \cdot 4) \\
19(2 \cdot 2) \\
15(3.0) \\
6(2 \cdot 1) \\
47(2 \cdot 3)\end{array}$ \\
\hline
\end{tabular}

†Percentage of each phage type.

NC: do not conform to designated phage types.

^Isolates of $S$ virchow from 1985-1990.

ranged from $8.4 \%$ for phage type 8 , the most common phage type identified, to $2 \cdot 1 \%$ for phage type 2 . For phage type $19,5 \cdot 5 \%$ of isolates were from blood culture. Previous studies have shown that multiple resistance, including resistance to ampicillin, chloramphenicol, and trimethoprim is common in this phage type. ${ }^{5}$

\section{Discussion}

In England and Wales the overall incidence of bloodstream invasion in non-typhoidal salmonellas isolated from humans and identified in the DEP in the 10 years 1981 to 1990 was less than $2 \%$. For bloodstream isolates, strains of $S$ enteritidis and $S$ typhimurium were the most numerous, but this reflected the overall preponderance of these two serotypes over the study period. In terms of the number of bloodstream isolates/isolates received, however, the highest incidence of septicaemias $(74 \%)$ was in infections caused by $S$ cholerae-suis. This serotype has been associated with extra-intestinal infections in both the United States of America and Britain. $^{67}$ Fortunately, $S$ cholerae-suis is extremely uncommon in England and Wales, with strains each year accounting for less than 0.01 of isolations referred to the DEP. The second most invasive serotype was $S$ dublin, $25 \%$ of isolates of this serotype were from blood culture. Although $S$ dublin is not an important cause of food poisoning in England and Wales, the high incidence of septicaemia is of particular concern, and infections with this serotype need careful clinical supervision.

$S$ dublin was the second most common serotype in cattle for the 12 year period 197889 , and $S$ cholerae-suis ranked in the top 10 of serotypes in pigs. ${ }^{8}$ It is interesting to note that although $S$ dublin and $S$ cholerae-suis are rarely isolated from humans, a particular feature of human infections with these "species-specific" serotypes is the high incidence of bloodstream invasion.

Other salmonellas with septicaemia rates greater than $2 \%$ included $S$ virchow, $S$ heidelberg, $S$ panama, $S$ kedougou, $S$ bovismorbificans and $S$ ealing. $S$ virchow was the third most common serotype in humans over the 10 year period. A high incidence of blood stream isolations of $S$ virchow has been observed in outbreaks in Liverpool, ${ }^{9}$ Manchester, ${ }^{10}$ and Scotland. ${ }^{11} S$ heidelberg and $S$ panama have ranked in the top 10 of serotypes identified in the DEP each year from 1981 through to 1990 and have each comprised about $1 \%$ of the total isolates. Since $1989 S$ kedougou has become an increasingly important cause of food poisoning in England and Wales ${ }^{12}$ and in 1989 was responsible for a series of outbreaks associated with cooked turkey meat. $S$ ealing and $S$ bovis-morbificans have only been rarely isolated from cases of food poisoning in England and Wales. In late 1985, however, $S$ ealing associated with a contaminated dried milk product caused an outbreak among infants. ${ }^{13}$ Two of 54 isolates from infants infected in this outbreak were from blood culture. In a study of strains isolated in Liverpool from 1969-1984, $S$ london was reported to cause a higher than average number of extra-intestinal infections, ${ }^{7}$ but in this investigation only one of 95 isolates of this serotype was from blood culture.

For $S$ typhimurium phage types there was little overall difference in the incidence of blood stream isolations. It is noteworthy, however, that $2.4 \%$ of phage type $204 \mathrm{c}$ isolates were from blood culture, and because strains of this phage type are invariably resistant to at least four antimicrobial drugs, ${ }^{5}$ the choice of treatment may be restricted in cases of sepicaemia. Likewise, $5.5 \%$ of isolates of $S$ virchow phage type 19 were from the bloodstream, and like $S$ typhimurium phage type 204c, most isolates of this phage type have been reported to be multiresistant. ${ }^{5}$

These results show that in England and Wales most salmonella serotypes seldom cause septicaemia. It is particularly reassuring that the incidence of bloodstream invasion in the two most common serotypes, $S$ enteritidis and $S$ typhimurium, is less than $2 \cdot 0 \%$.

1 Kauffmann F. Serological diagnosis of Salmonella species. Copenhagen: Munksgaard E, 1972.

2 Callow. B. A new phage typing scheme for Salmonella typhimurium. J Hyg 1959;57:346-59.

3 Ward LR, de Sa JDH, Rowe B. A phage-typing scheme for Salmonella enteritidis. Epidemiol Infect 1988;99:291-4. 4 Chambers RM, McAdam P, de Sa JDH, Ward LR, Rowe B. 
A phage-typing scheme for Salmonella virchow. FEMS 155-7.

5 Ward LR, Threlfall EJ, Rowe B. Multiple drug resistance in salmonellae isolated from humans in England and Wales: comparison of 1981 with 1988. J Clin Pathol 1990;43: 563-6.

6 Saphra I, Wasserman M. Salmonella cholerae-suis: clinical and epidemiological evaluation of 329 infections identified between 1940 and 1954 in the New York Salmonella Center. Am J Med Sci 1954;228:523-5.

7 Wilkins EGL, Roberts C. Extraintestinal salmonellosis. Epidemiol Infect 1988;100:361-8.

8 Anonymous. Animal Salmonellosis 1989. Ministry of Agriculture, Fisheries and Food; Welsh Office. DepartAgriculture, Fisheries and Food; Welsh Office. Depa
ment of Agriculture and Fisheries for Scotland. 1991.
9 Semple AB, Turner GC, Lowry DMO. Outbreak of foodpoisoning caused by Salmonella virchow in spit-roasted chicken. Br Med J 1968;ii:801-3.

10 Mani V, Brennand J, Mandal BK. Invasive illness with Salmonella virchow infections. Br Med J 1974;i:143-4.

11 Todd WTA, Murdoch JM. Salmonella virchow: a cause of significant bloodstream invasion. Scott Med J 1983; 28:176-8.

12 Threlfal EJ, Hall MLM, Frost JA, Rowe B. A plasmid typing scheme for Salmonella kedougou. In: Proceedings of the 2nd International Meeting on Bacteriological Epidemiological Markers. Athens: Ascent Ltd, 1990;171.

13 Rowe B, Hutchinson DN, Gilbert RJ, et al. Salmonella ealing infections associated with consumption of infant ealing infections associated with 